\title{
The fitness costs and trade-off shapes associated with the exclusion of nine antibiotics by OmpF porin channels
}

\author{
Katherine Phan and Thomas Ferenci \\ School of Life and Environmental Sciences, University of Sydney, Sydney, New South Wales, Australia
}

\begin{abstract}
The trade-off relationship between antibiotic exclusion and nutrient access across the Gram-negative outer membrane is determined by structural constraints in porin channels. The precise nutritional cost of exclusion is unknown for different antibiotics, as are the shapes of the nutrition-susceptibility trade-off. Using a library of 10 engineered isogenic Escherichia coli strains with structural modifications of OmpF porin expressed at a constant level, susceptibilities were measured for nine antibiotics and the nutritional fitness costs estimated by competitions in chemostats. Different antibiotics exhibited a remarkably varied range of geometries in the nutrition-susceptibility trade-off, including convex, concave and sigmoidal trade-off shapes. The trade-off patterns predict the possibility of adaptations in contributing to antibiotic resistance; exclusion of amoxicillin or trimethoprim in ompF mutants can occur with little loss of fitness whereas kanamycin and streptomycin exclusion has a high cost. Some individual OmpF changes even allow positive correlations (trade-ups), resulting in increased fitness and decreased susceptibility specifically to cephalexin or ciprofloxacin. The surprising plasticity of the nutrition-exclusion relationship means that there are no generalisable rules that apply to decreasing susceptibility for all antibiotics. The protein changes are exquisitely specific in determining nutritional fitness and adaptive outcomes in a structural constraint trade-off.

The ISME Journal (2017) 11, 1472-1482; doi:10.1038/ismej.2016.202; published online 10 January 2017
\end{abstract}

\section{Introduction}

Negative links between traits, or trade-offs, are longobserved in a variety of organisms (Stearns, 1989). Trade-offs are ecologically significant and have been used to explain the coexistence of organisms and evolutionary diversification and speciation (Tilman, 2000; Kneitel and Chase, 2004). Bacteria provide simple models to study trade-offs without multicellular interactions and temporal changes during life cycles (Bohannan et al., 2002; Jessup et al., 2005; Gudelj et al., 2010). Trade-offs are common in bacteria (Ferenci, 2016) and here we focus on the molecular basis of a bacterial trade-off between survival (antibiotic exclusion) and multiplication (access to nutrients).

A key characteristic of trade-offs is the geometry of the quantitative relationship between traits such as antibiotic exclusion and growth fitness. The shape of trade-offs was identified as being extremely important in predicting the behaviour of organisms

Correspondence: T Ferenci, School of Life and Environmental Sciences, University of Sydney, Building G08, Sydney, New South Wales 2006, Australia.

E-mail: tom.ferenci@sydney.edu.au

Received 30 August 2016; revised 20 November 2016; accepted 1 December 2016; published online 10 January 2017
(Levins, 1968; Maharjan et al., 2013). Especially relevant here is that the geometry of trade-offs can explain the evolution of organisms as specialists or generalists (Levins, 1968). For example, the shape can determine whether antibiotic resistance can be developed with or without fitness costs (that is, as generalists). Negative correlations can form linear convex, concave or sigmoidal curves (Maharjan et al., 2013). Costs can accelerate (increase quicker than the benefits; concave shape) or decelerate (increase slower than the benefits; convex shape (Hoyle et al., 2008)). In our current example, the shape should distinguish whether antibiotic exclusion is likely to incur accelerating or decelerating costs and consequently predict the adaptive behaviour of antibiotic resistant organisms.

Defining precise trade-off shapes is difficult because of the heterogeneity of random mutations and natural populations. Trade-offs are rarely studied with clean genetic backgrounds or fixed levels of expressions of traits. The process is further complicated because the shape can change when pairs of traits (such as phage resistance and nutrition or stress resistance and nutrition) are measured in different environments (Jessup and Bohannan, 2008; Maharjan et al., 2013). It was recently proposed that trade-off geometry can be deduced from fundamental 
properties of bacteria and used to predict trade-off shapes (Meyer et al., 2015), but the link(s) between mechanism and geometry still need a great deal of exploration.

In bacteria, three distinct underlying molecular mechanisms were proposed to explain 30 pairs of traits that have some evidence for negative correlation (Ferenci, 2016). These mechanisms can be defined as resource allocation, structural constraint and informational processing trade-offs (Ferenci, 2016). It is unknown as yet whether the underlying mechanism has an influence on trade-off shape. One class of trade-off, based on resource allocation, has been studied in detail (Maharjan et al., 2013). Here we deal with a mechanistically different structural constraint trade-off and delve into how structural modification changes traits and trade-off geometries. Specifically, we describe in detail how amino acid changes in a porin protein reciprocally affect permeability for nutrients and antibiotic exclusion.

The traits we study are shaped by the intrinsic permeability barrier known as the outer membrane in Gram-negative bacteria (Nikaido, 2003; Delcour, 2009; Masi and Pagès, 2013). Nutrients such as mono- and di-saccharides and amino acids cross the outer membrane through water-filled protein channels known as porins. Many classes of antibiotics also use this polar route to cross the outer membrane of bacteria (Pages et al., 2008; Delcour, 2009; Ferenci and Phan, 2015). Access of nutrients and antibiotics is controlled by the pore size in general porin proteins like $\mathrm{OmpF}$ and $\mathrm{OmpC}$ in Escherichia coli, where optimal nutrient access is favoured by the larger porin channel OmpF. Nutrient uptake can also be increased through the use of additional soluteselective proteins like LamB glycoporin in E. coli (Death et al., 1993; Liu and Ferenci, 1998) or through several Pseudomonas aeruginosa porins like OprB and OprD (Wylie and Worobec, 1994; Ochs et al., 1999). Porins contribute to a nutrition-stress protection trade-off (Ferenci, 2005) in that high nonspecific outer membrane permeability is good for nutrition but a liability in unfavourable circumstances, such as with antibiotics.

The antibiotics we chose to study all have evidence that porins are involved in their permeation. These include quinolones and $\beta$-lactams (Sawai et al., 1992), chloramphenicol (Mortimer and Piddok, 1993), trimethoprim (Fernández and Hancock, 2012) and aminoglycosides (Nakae and Nakae, 1982). The case of aminoglycosides is interesting because the passage of some like kanamycin through porins has been questioned (Hancock et al., 1991a). Our studies here address this question.

Although it should be noted that antibiotic susceptibility is affected by other mechanisms such as efflux, mutations affecting porins contribute to the armory of emerging superbugs and resistance to several important antibiotics (Pages et al., 2008; Nikaido, 2009). Porin changes are involved in the multi-drug resistance problem in Gram-negative bacterial infections (Pages et al., 2008; Ferenci and Phan, 2015). In this communication, we consider the fitness implications of changes in porins when permeability either needs to decrease (antibiotic exposure) or increase (under extreme nutrient limitation). Piecemeal information on permeability in porin mutants is available (Jeanteur et al., 1994; Zhang and Ferenci, 1999; Simonet et al., 2000; Phale et al., 2001), so we chose to analyse the trade-off changes shown in Figure 1 with eight structural modifications. We describe experiments to test how sequence changes in porins affect nutrition and resistance in order to define the molecular basis of

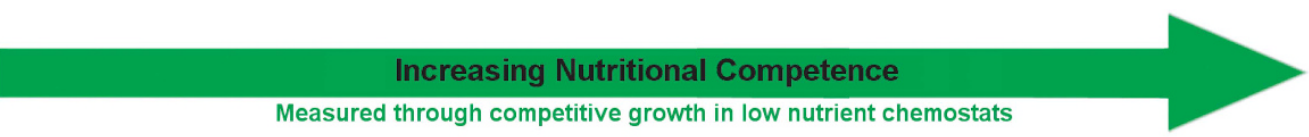

a

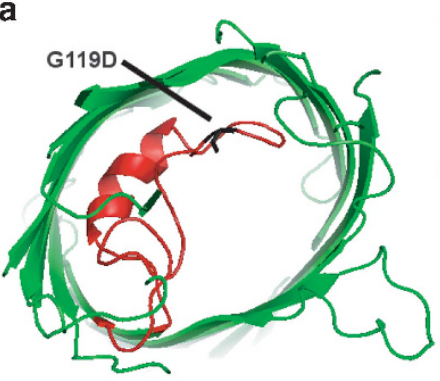

b

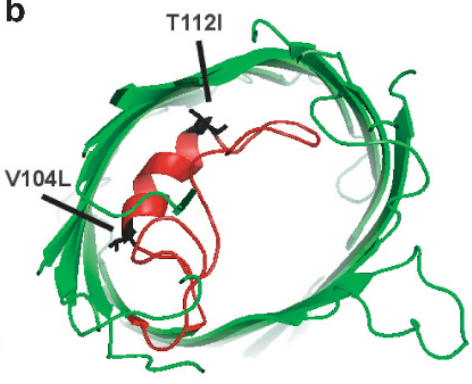

C

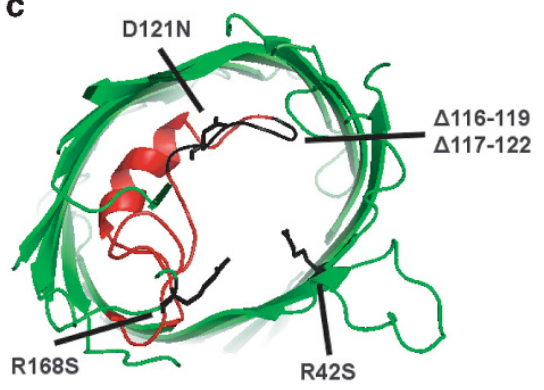

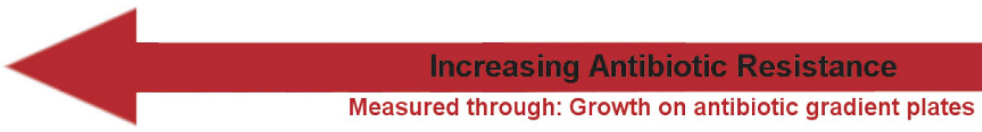

Figure $1 \mathrm{OmpF}$ porin structural variations. These are shown in a cross section of the constriction zone of an OmpF monomer (PDB file $2 \mathrm{OMF}$ ), viewed from the extracellular side. The eye region is defined by the L3 constriction loop (red). Sites changed in this study are in black. (a) Substitution resulting in lower susceptibility to antibiotics than wild-type porin due to a constricted pore (Jeanteur et al., 1994). (b) Substitutions near-neutral in susceptibility. (c) Substitutions resulting in greater than wild-type susceptibility (Zhang and Ferenci, 1999). 
trade-off shapes under highly controlled conditions. Another related question we address is how stable these shapes are. After all, the predictive ability of trade-off shapes is valuable only if the shapes themselves are robust and insensitive to extrinsic factors. Our data actually indicate an unexpected level of plasticity in trade-off shapes with different antibiotics, which has important implications for trade-offs in general.

\section{Materials and methods}

Bacterial strains and strain constructions

Bacterial strains used in this study are listed in Table 1 and were variants of the wild-type E. coli K-12 strain MG1655 (Blattner et al., 1997). In the synthetic strains in Table 1 , the presence of the alternative porin OmpC was eliminated through transduction of ompC:: Tn10 into MG1655. The BW7000 strain was cured of tetracycline resistance via selection on chlortetracycline as described in (Maloy and Nunn, 1981). Wild-type OmpF was subsequenty inactivated through transduction of ompF::kan and replaced with variant ompF sequences as shown in Supplementary Figure S1. OmpF expression was controlled through the use of a synthetic promoter (its sequence and other primer sequences are shown in Supplementary Table S1) and elimination of post-transcriptional regulation. Transcriptional control was removed by replacing the upstream regulatory region with the drug resistance gene cat. The eight base pairs directly upstream of $o m p F$ were altered (A replaced with T and vice versa) to prevent post-transcriptional regulation by MicF and RybB. The artificial promoter was based on the RpoSleaderR4 sequence from Maharjan et al., 2013 and the synthetic region was inserted using the lambda red recombination system (Datsenko and Wanner, 2000; Supplementary Figure S1A). To generate the synthetic region upstream of $o m p F$, primer pair OmpFleaderF1/OmpFleaderR3 with pKD4 template was used. To aid recombination, $220 \mathrm{bp}$ homologous to asnS was added to the beginning of the PCR product using primers OmpFFExtenF1, OmpFFExtenR2 and OmpFFExtenR3. Mutant strains were selected as chloramphenicol and kanamycin resistant.

A multistep process was used to generate strains with the planned structural mutations (Supplementary Figure S1). PCR products containing a frameshift mutation in the cat gene and the desired structural mutation were generated as described in Supplementary Figure S1B. PCR products were inserted into BW7004 using lambda red recombination (Supplementary Figure S1) replacing ompF::kan and disrupting cat. The culture was grown overnight on $\mathrm{MMA}+0.2 \%$ (wt/vol) lactose and the larger colonies were screened for sensitivity to chloramphenicol and kanamycin. To generate isogenic strains with an antibiotic marker, for use in competition assays, strains resistant to chloramphenicol but sensitive to kanamycin were also selected.

\section{Culture conditions}

The growth medium used in all experiments unless otherwise stated was minimal medium A (MMA (Miller, 1972)) supplemented with $1 \mathrm{\mu g} \mathrm{ml}^{-1}$ thiamine and $1 \mathrm{mM} \mathrm{MgSO}_{4}$, and lactose at concentrations stated for each experiment. Luria-Bertani broth or MMA containing $0.2 \%(\mathrm{w} / \mathrm{v})$ sugar was used in batch culture.

For fitness measurements, chemostats were used as previously described (Phan and Ferenci, 2013). The chemostats were fed with $1 \times$ MMA supplemented with $0.02 \%$ (wt/vol) lactose, all at $0.3 \mathrm{~h}^{-1}$ dilution rate. The strains with $\mathrm{OmpF}$ structural changes were competed against BW7006 (with isogenic regulation controlling the wild-type $\mathrm{OmpF}$ sequence) or BW7014 (isogenic regulation with wildtype $\mathrm{OmpF}$ sequence but with chloramphenicol resistance) in chemostats. Competing strains were initially grown in independent chemostats at $37^{\circ} \mathrm{C}$ overnight. Cultures were mixed 50:50 in the same medium to start competition at the same dilution rate. The progress of the competition was monitored from samples taken at five time points over $24 \mathrm{~h}$.

Table 1 Strains used in this study

\begin{tabular}{|c|c|c|}
\hline Strain & Description & Origin or Reference \\
\hline MG1655 & Wild-type Escherichia coli K-12 & Blattner et al., 1997 \\
\hline BW7000 & MG1655 ompC::Tn10 & This study \\
\hline BW7001 & MG1655 ompC::Tn10(Tet $\left.{ }^{\mathrm{S}}\right)$ & This study \\
\hline BW7002 & MG1655 ompC::Tn10 $\left(\mathrm{Tet}^{\mathrm{S}}\right) \mathrm{cml}$ inserted into ompFleader & This study, see Supplementary Figure S1 \\
\hline BW7003 & MG1655 ompC::Tn10(Tet $\left.{ }^{\mathrm{S}}\right)$ ompF::kan & This study, see Supplementary Figure S1 \\
\hline BW7004 & MG1655 ompC::Tn10(Tet $\left.{ }^{\mathrm{S}}\right)$ ompFleader::cml ompF::kan & This study, see Supplementary Figure S1 \\
\hline BW7005 & BW7004 ompF(G119D) & Jeanteur et al., 1994 \\
\hline BW7006 & BW7004 ompF(replaced with WT ompF) & This study, see Supplementary Figure S1 \\
\hline BW7007 & BW7004 ompF(T112I) & This study, see Supplementary Figure S1 \\
\hline BW7008 & BW7004 ompF(V104L) & This study, see Supplementary Figure S1 \\
\hline BW7009 & BW7004 ompF(R168S) & Ziervogel and Roux, 2013 \\
\hline BW7010 & BW7004 ompF(D121N) & Benson et al., 1988, Saint et al., 1996 \\
\hline BW7011 & BW7004 ompF(R42S) & Ziervogel and Roux, 2013 \\
\hline BW7012 & BW7004 ompF( $1116-119)$ & Zhang and Ferenci, 1999 \\
\hline BW7013 & BW7004 ompF( $1117-122)$ & Zhang and Ferenci, 1999 \\
\hline
\end{tabular}


Bacteria in the mixed population were counted by serial dilution and plating onto L-agar (for total counts) or with $12.5 \mu \mathrm{g} \mathrm{ml}^{-1}$ chloramphenicol plates.

Antibiotic sensitivity was measured using the gradient plate technique described by (Szybalski and Bryson, 1952). Briefly, two layers of agar were set in a square $100 \mathrm{~mm}^{2}$ Petri dish (Sastedt, Technology Park, Adelaide, SA, Australia). The first layer contained $50 \mathrm{ml}$ LA and was set at an angle where the LA just covered the entire bottom of the Petri dish. After placing the Petri dish in a horizontal position, the second layer containing $50 \mathrm{ml}$ LA +antibiotic $\left(6 \mu \mathrm{g} \mathrm{ml}^{-1}\right.$ chloramphenicol, $0.3 \mu \mathrm{g} \mathrm{ml}^{-1}$ trimethoprim, $15 \mu \mathrm{g} \mathrm{ml}^{-1}$ streptomycin, $30 \mu \mathrm{g} \mathrm{ml}^{-1}$ kanamycin, $35 \mu \mathrm{g} \mathrm{ml}^{-1}$ spectinomycin, $3 \mu \mathrm{g} \mathrm{ml}^{-1}$ nalidixic acid, $0.03 \mu \mathrm{g} \mathrm{ml}^{-1}$ ciprofloxacin, $15 \mu \mathrm{g} \mathrm{ml}^{-1}$ cephalexin or $7 \mu \mathrm{g} \mathrm{ml}^{-1}$ ampicillin) was added and left to dry in a biosafety cabinet for $1 \mathrm{~h}$. The plate was subsequently left for $15 \mathrm{~h}$ to create the linear gradient by diffusion of antibiotic between the two layers. Strains were cross-streaked from areas of lower to higher antibiotic concentration and incubated overnight at $37^{\circ} \mathrm{C}$. Growth along streak lines was measured using a ruler.

The effect of efflux on antibiotic sensitivity was also determined through the use of gradient plates with the addition of the oxidative phospohorylation uncoupler, carbonyl cyanide 3-chlorophenylhydrazone (CCCP). Gradient plates were made and analysed as described above with the addition of $24 \mu \mathrm{M}$ CCCP to the agar layers.

Antibiotic sensitivity was also measured through altered growth rates in broth culture. Strains were grown to exponential phase at $37^{\circ} \mathrm{C}$ in LB. Optical density of the culture was measured and diluted to an inoculum of 100 bacteria. The strains were grown at $37^{\circ} \mathrm{C}$ with shaking in 96-well microtitre plates with each well containing $100 \mu \mathrm{l}$ LB+antibiotic ( $0.5 \mu \mathrm{g} \mathrm{ml}^{-1}$ chloramphenicol, $0.1 \mu \mathrm{g} \mathrm{ml}^{-1}$ trimethoprim, $6.5 \mu \mathrm{g} \mathrm{ml}^{-1}$ streptomycin, $17 \mu \mathrm{g} \mathrm{ml}^{-1}$ kanamycin, $22 \mu \mathrm{g} \mathrm{ml}^{-1}$ spectinomycin, $3 \mu \mathrm{g} \mathrm{ml}^{-1}$ nalidixic acid, $\quad 0.005 \mu \mathrm{g} \mathrm{ml}^{-1}$ ciprofloxacin, $8.25 \mu \mathrm{g} \mathrm{ml}^{-1}$ cephalexin or $2 \mu \mathrm{g} \mathrm{ml}^{-1}$ ampicillin). Growth was measured for $8 \mathrm{~h}$ with readings taken every $15 \mathrm{~min}$ in a Tecan Infinite M1000Pro Plate reader.

\section{OmpF quantitation}

OmpF expression was measured using an immunoassay based on (Death et al., 1993). Briefly, strains were grown to exponential phase in LB of differing $\mathrm{NaCl}$ concentration $\left(0 \mathrm{gl}^{-1}, 1.25 \mathrm{~g} \mathrm{l}^{-1}, \quad 2.5 \mathrm{gl}^{-1}\right.$, $5 \mathrm{gl}^{-1}, 10 \mathrm{gl}^{-1}$ and $20 \mathrm{gl}^{-1}$ ) then washed and resuspended in saline to a density of $2 \times 10^{8}$ cells per ml. Two microliter of bacterial suspension was applied to nitrocellulose membrane (GE Healthcare, Parramatta, NSW, Australia) under gentle suction and air dried for $10 \mathrm{~min}$. Additional protein sites were blocked using saline $+5 \%$ skim milk powder for $15 \mathrm{~min}$ then incubated with 1:5000 diluted antiOmpF antibody (donated by Prof $\mathrm{P}$ Reeves) for $15 \mathrm{~min}$. The membrane was washed with saline then incubated with 1:1000 diluted enzyme (anti-rabbit IgG horseradish peroxidase (Amersham, Little Chalfont, UK) for $15 \mathrm{~min}$. The membrane was washed with saline then incubated with ECL solution (GE Healthcare) and visualised on film (GE Healthcare).

\section{Data analysis}

The comparison of antibiotic susceptibility assays was done by rank ordering. The strain set members were ranked for each antibiotic from most to least resistant. Ranking for the gradient plate assay was determined by distance of growth along the streak line, where longer growth was ranked more resistant. Ranking for the broth culture assay for all antibiotics except for amoxicillin was determined by comparison of growth rates in antibiotic broth to growth rate in LB, where a lesser change in growth rate was ranked as more resistant. Spearman's rank order correlation was determined using the correlation function in GraphPad Prism version 7 (GraphPad Software. San Diego, CA, USA).

Curve fitting and analysis of trade-off shapes was performed using GraphPad Prism version 7. Nonlinear regression was used to generate linear, quadratic and sigmoidal curves and Akaike's information criterion and $R^{2}$ values used to determine the bestfitting curve.

Statistical analysis of differences in growth rates or selection co-efficients was performed in Microsoft Excel. The F-test was used to determine whether variance between compared strains was equal and the appropriate two-sample two-tailed $t$-test (assuming equal or unequal variance) applied.

\section{Results}

The strain set with fixed expression of ompF but varying in $\mathrm{OmpF}$ structure

It has been previously shown that the effects of structural changes on trade-off shapes can be masked by other factors such as variation in porin levels (Phan and Ferenci, 2013). In order to analyse the implications of a structural constraint trade-off, the focus of this study is on traits dependent purely on $\mathrm{OmpF}$ functionality. This focus on structure can be achieved only if a number of complicating factors are eliminated in the synthetic strain set used (listed in Table 1). These included genetically inactivating a second general porin, OmpC, in the strains as well as disruption of the regulation that makes the cellular level of OmpF sensitive to environmental variations (Pratt et al., 1996). To avoid changes in porin levels with nutrient depletion (Liu and Ferenci, 1998) or the presence of antibiotics (Dupont et al., 2007; Lin et al., 2012), the transcriptional and translational regulation of $\mathrm{ompF}$ was altered through the replacement of the promoter-leader region by a synthetic promoter upstream of ompF (see Supplementary Figure S1 for details). This new synthetic promoter removed the OmpR/EnvZ and CpxR regulation of 
$o m p F$ as well as the translational control through MicF (De la Cruz and Calva, 2010), all of which are important in environmental regulation. The synthetic promoter was demonstrated to maintain constant levels of OmpF protein under conditions changing wild-type regulation (see blot data in Supplementary Figure S2). The fixed amount of OmpF produced from the artificial promoter was in the range seen in the E. coli ECOR strain collection (Phan and Ferenci, 2013) and within the range of OmpF levels expressed between high- and lowsalt conditions in E. coli K-12 (Supplementary Figure S2).

The structural changes shown in Figure 1 were introduced using site-directed mutagenesis of ompF into strains with the artificial promoter. The mutations were transferred into BW7004 (ompF::kan mutant) using lambda red recombination (Supplementary Figure S1). Seven structural changes were chosen from the literature based on altered permeability of various solutes. One substitution, G119D (Figure 1a), was known to decrease OmpF permeability through an interaction of residues dividing the OmpF channel into two smaller channels (Jeanteur et al., 1994). Two changes, $\Delta 116-119$ and $\Delta$ 117-123 (Figure 1c), increased channel permeability for sugars by deleting part of the L3 constriction loop (Zhang and Ferenci, 1999), thereby increasing channel size. Three substitutions, D121N, R168S and R42S (Figure 1c), were due to point mutations known to increase sensitivity to antibiotics (Benson et al., 1988; Saint et al., 1996; Ziervogel and Roux, 2013). The final two changes, T112I and V104L (Figure 1b), were caused by random point mutations affecting the L3 loop that occurred due to errors in the PCR process. All the $\mathrm{OmpF}$ variants grew on minimal media with lactose in batch culture. Exponential lactose growth rates with the synthetic $\mathrm{OmpF}$ proteins were similar to wild-type $\mathrm{OmpF}$ (indistinguishable in two-sample two-tailed $t$-tests (all $P$-values $>0.06$ )) but all were faster than in the porinless mutant (two-sample two-tailed $t$-tests (all $P$-values $<0.04)$ ), indicating that they contributed to the permeability of sugar.

The effect of porin structure on nutrient permeability Porin is especially important at limiting nutrient levels (Liu and Ferenci, 1998) so growth in batch culture with excess nutrient is not fully indicative of the fitness implications of porin channel changes. A sensitive differentiation of porin-dependent variation in nutrient access was made through measuring fitness when growing on limiting amounts of lactose in a chemostat environment. Lactose is ideal for this purpose because it permeates primarily through OmpF (Zhang and Ferenci, 1999) and it is not a substrate for the LamB glycoporin (Death et al., 1993). The fitness measurement in Figure 2 was conducted through competition of each mutant against the isogenic strain containing wild-type

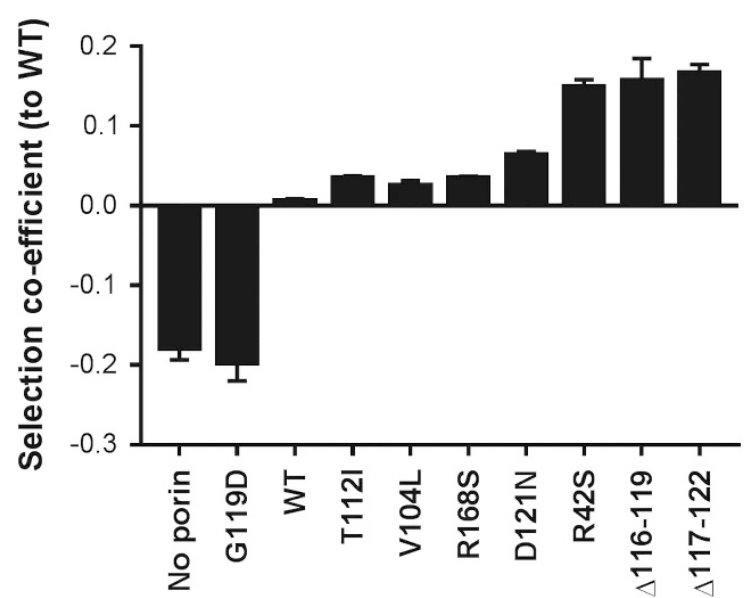

Figure 2 Fitness effects of porin changes at low nutrient levels. The selection co-efficient of the OmpF variant strains is shown when competed against a strain with wild-type porin in chemostats limited for lactose as nutrient $(0.02 \%$ lactose in the feed medium). Population changes at a specific growth rate of $0.3 \mathrm{~h}^{-1}$ were sampled over $24 \mathrm{~h}$. The competitions were replicated twice and error bars show s.d.

$\mathrm{OmpF}$ at the same protein level as in the other constructs. To compare strains, the changes in the proportion of wild-type and mutant in the mixed population over the initial linear portion of the competition curve was converted into a selection coefficient, $s$ (Dykhuizen and Hartl, 1983). The selection co-efficient is positive if the structural mutation resulted in a strain fitter on lactose than wild-type, whereas structural mutations resulting in less fit strains had a negative $s$ value (Figure 2).

As shown in Figure 2, OmpF structure has a huge effect on fitness in lactose-limited chemostats. The strain set members show a broad range of fitness properties depending on the sequence changes. Two strains in the strain set, those with no porin and with the G119D change, strongly decreased strain fitness relative to wild-type protein (two-sample two-tailed $t$-test $P$-values $<0.002$ and $<0.0005$, respectively). This is consistent with reduced nutrient access in a porinless strain (von Meyenburg, 1971) and with a reduction in permeability with the G119D constriction of the channel (Jeanteur et al., 1994). Three structural mutations, R42S, $\Delta 116-119$ and $\Delta 117-$ 123, were found to significantly increase strain fitness (two-sample two-tailed $t$-test $P$-values: $<0.0000001,<0.001$ and $<0.0002$, respectively). The $\Delta 116-119$ and $\Delta 117-123$ deletions remove several residues of the OmpF L3 constriction loop (Figure 1), which allows lactose to permeate more rapidly. The T112I, V104L, R168S and D121N substitutions increase fitness slightly but significantly (two-sample two-tailed $t$-test $P$-values all $<0.01$ ). Interestingly, the R42S substitution had a large fitness increase comparable to the L3 deletion mutants. Kojima and Nikaido (2013) showed that some point mutations changing charged residues in porins lead to altered permeability. The acidic 
residues on the L3 loop face basic residues on the opposite beta-barrel wall and the G119D, R42S or R168S substitutions may change the strong electric field inside the pore with different effects on lactose access (and antibiotic access as seen below).

The effect of porin structure changes on antibiotic exclusion

The role of OmpF structure in antibiotic exclusion was measured on agar plates containing set gradients of antibiotic concentration (Figure 3a). The concentration gradient used in the plates was determined from preliminary experiments to give a range of growth effects as shown in Figure 3. Of the nine antibiotics tested, three (streptomycin, kanamycin and spectinomycin) were aminoglycosides, two (cephalexin and amoxicillin) were $\beta$-lactams and the remaining four were examples of quinolones (nalidixic acid), fluroquinolones (ciprofloxacin), inhibitors of protein synthesis (chloramphenicol) and dihydrofolate reductase (trimethoprim). It should be noted that because of the
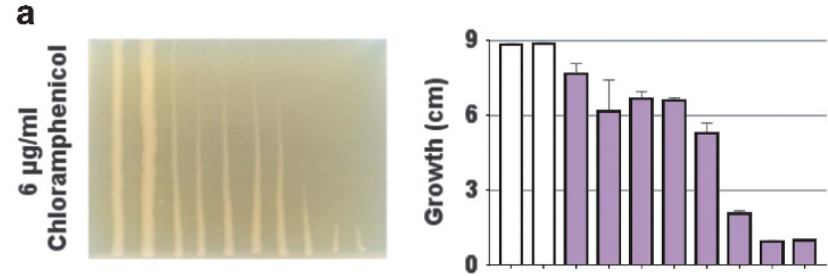

b
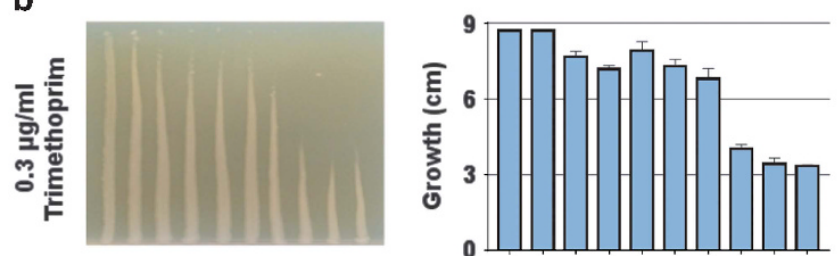

C
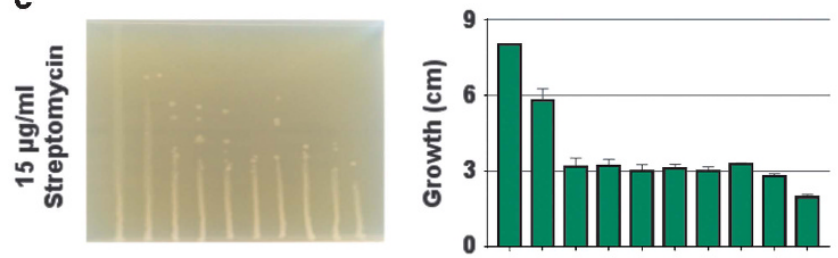

d
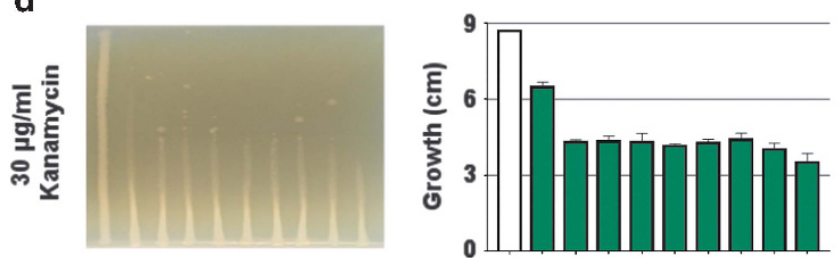

e
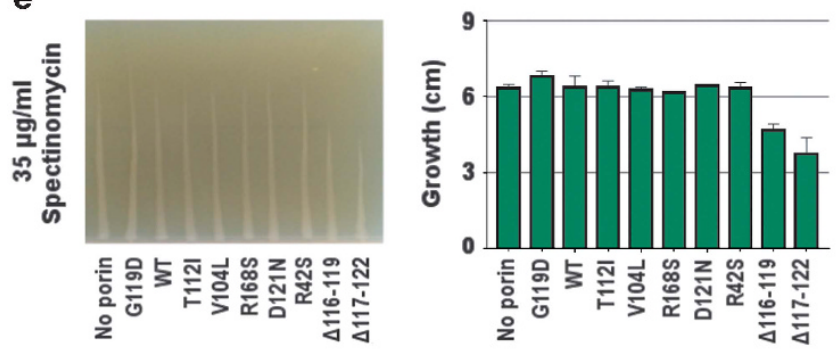

f
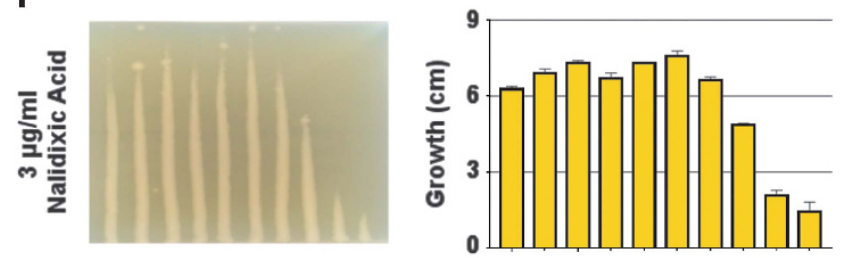

g
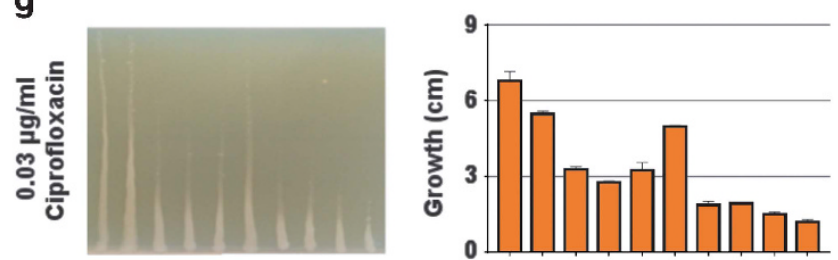

h
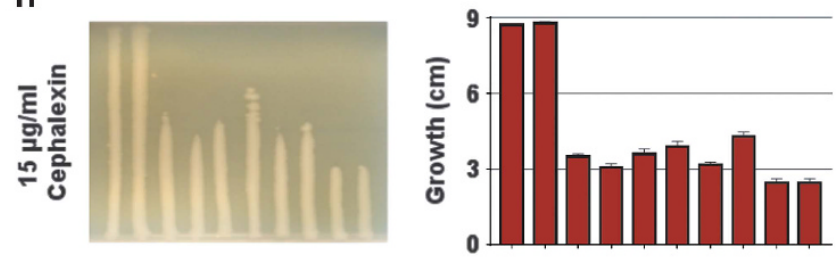

i
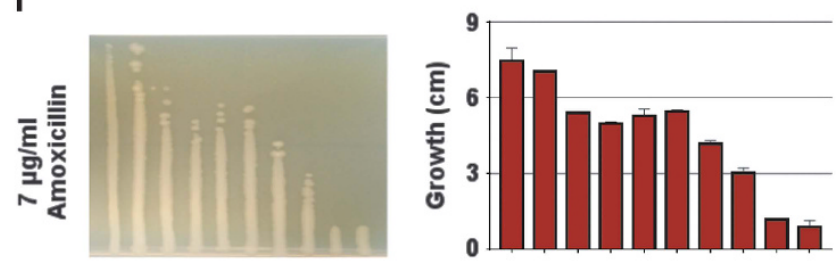

j

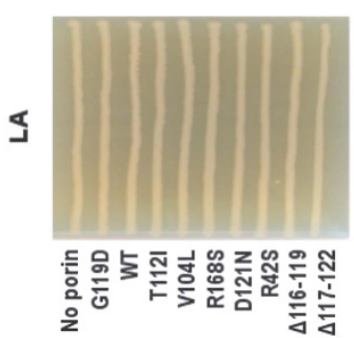

Figure 3 Antibiotic susceptibility of the $\mathrm{OmpF}$ strain set. The effects of antibiotics in a gradient plate are shown for: (a) $0-6 \mu \mathrm{mg} \mathrm{m}^{-1}$ chloramphenicol, (b) $0-0.3 \mu \mathrm{g} \mathrm{ml}^{-1}$ trimethoprim, (c) $0-15 \mu \mathrm{g} \mathrm{ml}^{-1}$ streptomycin, (d) $0-30 \mu \mathrm{g} \mathrm{ml} \mathrm{l}^{-1} \mathrm{kanamycin}^{-(\mathbf{e})} 0-35 \mu \mathrm{g} \mathrm{ml}{ }^{-1}$ spectinomycin, (f) $0-3 \mu \mathrm{g} \mathrm{ml}{ }^{-1}$ nalidixic acid, (g) $0-0.03 \mu \mathrm{g} \mathrm{ml}^{-1}$ ciprofloxacin, (h) $0-15 \mu \mathrm{g} \mathrm{ml}^{-1}$ cephalexin, (i) $0-7 \mu \mathrm{g} \mathrm{ml}{ }^{-1}$ amoxicillin and (j) a no antibiotic control. The white bars are results due to resistance genes in the strains tested as described in Supplementary Table S1. The growth on the gradient plate was measured in triplicate at the previously stated concentrations and the mean and standard deviation shown. 
construction steps in Supplementary Figure S1, BW7004 (the porinless mutant) contained resistance genes for chloramphenicol and kanamycin and BW7005 (G118D mutation) contained a resistance gene for chloramphenicol so were fully resistant in the gradient plates to these antibiotics.

For every antibiotic tested, access was porin channel dependent and susceptibility was affected in one or more ompF mutant. The strain order in Figure 3 is presented in line with their increasing fitness in Figure 2. The inhibition patterns for chloramphenicol, trimethoprim, ciprofloxacin and amoxicillin were similar, showing a continuum of resistance levels in different strains, from complete resistance to high susceptibility (Figure 3a). Another pattern was seen with streptomycin, kanamycin and cephalexin, which were excluded by only two OmpF variants (porinless and G119D). Spectinomycin and nalidixic acid were different from the others in that there was some susceptibility even in the porin-

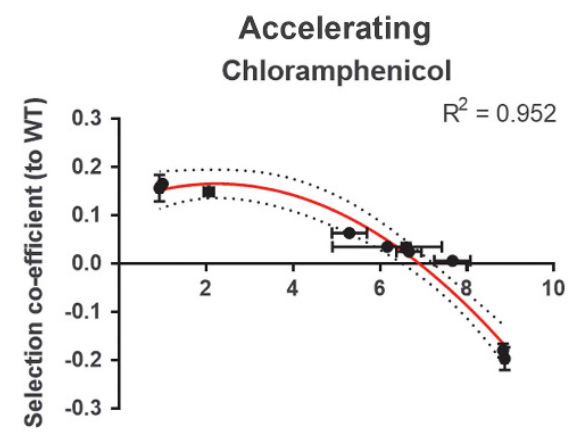

Amoxicillin

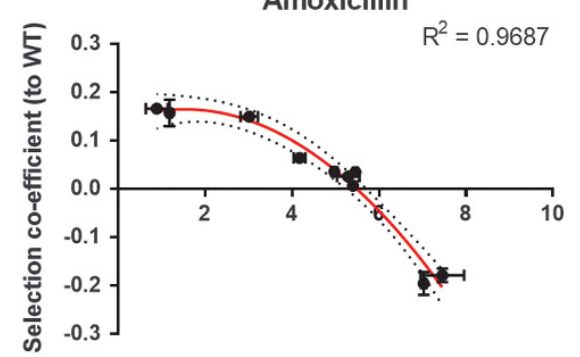

Trimethoprim

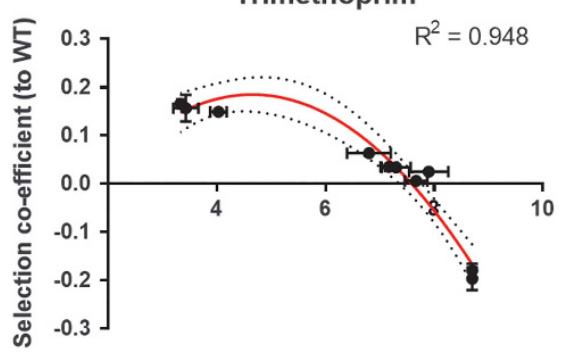

Spectinomycin

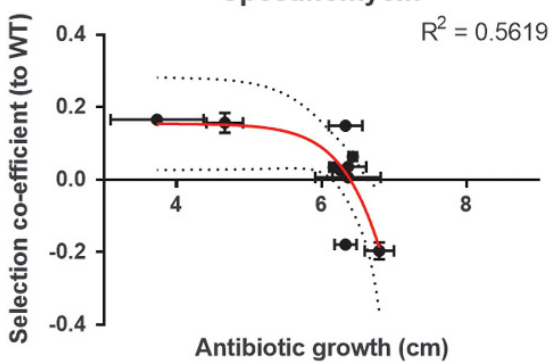

Linear

Nalidixic Acid

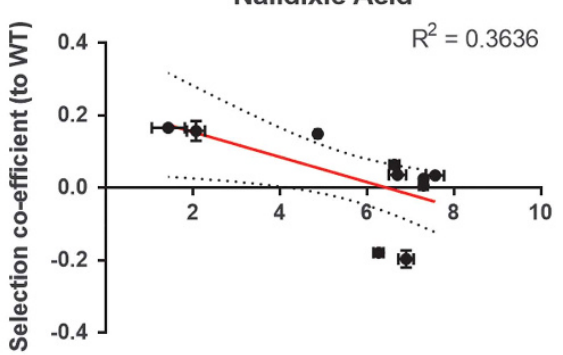

Ciprofloxicin
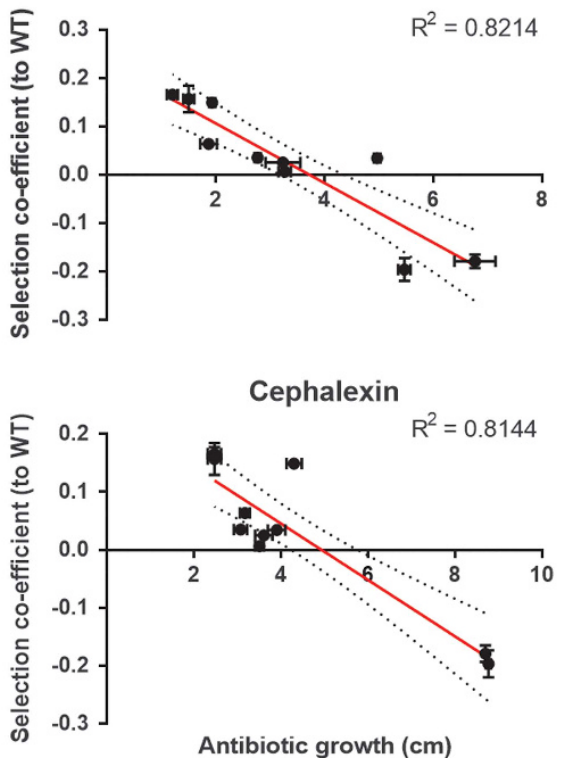

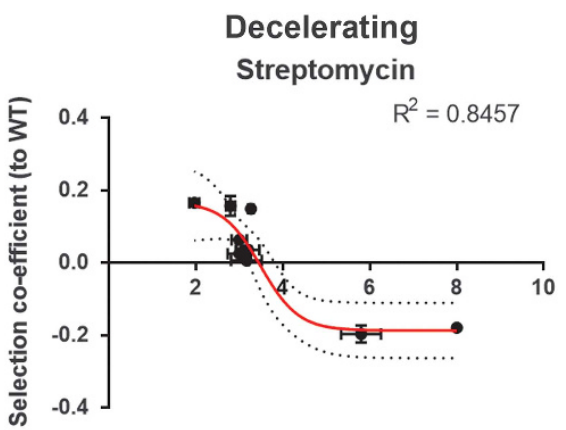

Kanamycin

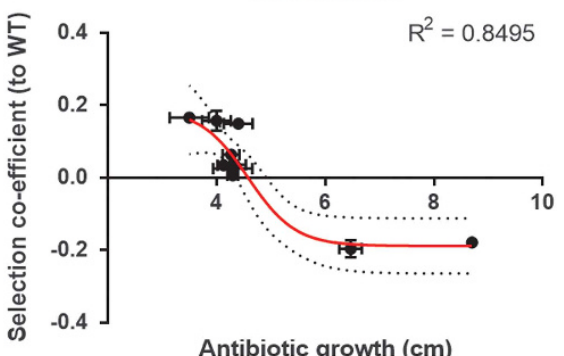

Antibiotic growth $(\mathrm{cm})$

Figure 4 The trade-off between nutrition and antibiotic susceptibility with 9 antibiotics. Each point represents the measured traits of a member of the OmpF strain set, with the $y$ axis representing fitness (selection co-efficient) and the $x$ axis showing antibiotic susceptibility in the gradient plates. The curve fitting to these points was determined using the linear regression analysis for linear, quadratic and sigmoidal curves (GraphPad Prism 7). The best fit curve (red line) was determined using AICc and $R^{2}$ values. These AICcs are tabulated in Supplementary Table S2A for all three models and all nine antibiotics. 95\% confidence limits (dotted lines) and $R^{2}$ values are shown. Error bars show the standard deviation for antibiotic susceptibility ( $x$ axis) or selection coefficient ( $y$ axis) based on data in Figures 2 and 3. 
less mutant, indicating non-OmpF dependent paths though the outer membrane. However, the increase in susceptibility by variants which greatly increased channel size ( $\Delta 116-119$ and $\Delta 117-123$ mutants), indicates these two antibiotics can also involve OmpF in passing through the outer membrane.

Interestingly, the resistance patterns of particular strains followed the same trend, that is, resistant strains were more resistant to all antibiotics and sensitive strains were more sensitive to all antibiotics in generally the same order. However, the R168S and R42S substitutions were observed to break this pattern. These mutations, which increase sensitivity to most antibiotics, were found to have a significantly increased resistance to ciprofloxacin and cephalexin relative to wild-type (two-sample two-tailed $t$-test $P$-values $<0.00002$ and $<0.002$, respectively).

To corroborate the gradient plate results, antibiotic effects were also determined from growth rates in batch culture (Supplementary Figure S3). For the majority of antibiotics growth rate was decreased in the sensitive strains. However, amoxicillin had a distinct growth rate pattern, as strains grew at the same rate, but reached stationary/death phase at different culture densities. Lower growth yields were also found to occur in a distinct manner with the majority of strains reaching stationary phase at a unique concentration.

Strains were ranked according to their level of antibiotic resistance (most to least resistant) in both gradient plates and broth culture. When the ranking was compared between the gradient plate and liquid culture techniques using Spearman correlations (Supplementary Figure S3), the strains exhibited a high degree of parallelism in antibiotic response in both methods. In the case of kanamycin, the only antibiotic that did not have a high degree of correlation, the problem was that the strains exhibited little differentiation in antibiotic susceptibility in both tests. Overall the effects on strains were consistent between gradient plates and growth rate experiments.

The shapes of the trade-offs between fitness and antibiotic exclusion by porins

The data in Figures 2 and 3 suggest negative correlations between fitness and exclusion; strains that are the fittest (most permeable to lactose) generally show the greatest susceptibility to all antibiotics tested. The shapes of the trade-offs shown in Figure 4 for each antibiotic provide some surprises however. The best fit for three antibiotics (nalidixic acid, ciprofloxacin and cephalexin) indicates a linear trade-off. Four antibiotics (chloramphenicol, amoxicillin, trimethoprim and spectinomycin) of very different structures and modes of action show accelerating fitness costs with decreasing susceptibility. The remaining two antibiotics, both aminoglycosides, show sigmoidal trade-off geometries with decelerating costs.
The effect of efflux on the growth- susceptibility tradeoff in the OmpF structural mutants

The susceptibility of bacteria to toxic molecules is decreased by efflux as well as porin exclusion (Pages et al., 2008; Kojima and Nikaido, 2013). To test if efflux influences the pattern in Figures 3 and 4, we additionally looked at antibiotic susceptibility in the presence of CCCP, an inhibitor of efflux energisation, at a concentration previously used to inhibit efflux with other E. coli strains (Moreira et al., 2005). As shown in Supplementary Figure S4, the major effect of CCCP on susceptibility is in the highly permeable strains, which become fully susceptible when efflux is inhibited. The other strains in the set are less affected by CCCP. The trade-off shapes in Figure 4 are also largely unchanged by CCCP so are indeed mainly influenced by OmpF structures (Supplementary Figure S5). For spectinomycin, which had the only different trade-off shape between Figure 4 and Supplementary Figure S5, the fitting of the points is the main variable and $R^{2}$ values are low. Thus efflux is not a major influence in determining the trade-off, which is constrained mainly by porin structure in Figure 4 and Supplementary Figure S5.

The overall conclusion from the results is that a structural constraint trade-off within a single protein does not produce the same trade-off shape with differing environments. Further variation of shapes within structural classes of antibiotic suggests that chemical structure does not constrain trade-off shapes either. The surprising plasticity of shapes in Figure 4 and Supplementary Figure S5 has major implications for the interpretation the costs of antibiotic resistance in terms of trade-offs, as discussed below.

\section{Discussion}

Our results provide a new perspective on trade-offs in antibiotic exclusion and the fitness implications of porin changes in drug-resistant bacteria. The use of the synthetic strain set varying only in $\mathrm{OmpF}$ porin in near-isogenic strains permitted trade-off geometries to be determined, without the heterogeneity and noise seen in less controlled studies of bacterial trade-offs (for example, Jessup and Bohannan, 2008; Phan and Ferenci, 2013). The strain set approach has allowed us to reveal well-defined trade-off shapes and the nutritional costs of changing the permeability of 9 commonly used antibiotics. The focus on structural variations has also allowed for the first time an analysis of a mechanistically defined structural constraint trade-off (Ferenci, 2016) in unprecedented detail. Most significantly, the variation of OmpF channel properties through amino acid changes with fixed expression levels revealed the multiple shapes resulting from the role of a porin in balancing nutrient uptake and antibiotic exclusion.

The competitive impact of OmpF structure on nutrient uptake has been quantified for the first time 
(Figure 2). The nature of the porin channel has a very large effect on growth fitness at limiting sugar levels. The selection coefficients in Figure 2 varied from +0.2 to -0.2 , depending on the mutation. As expected, large deletions in the L3 loop create the biggest increase in fitness at limiting sugar concentration; these mutations are indeed selected when bacteria are evolving in prolonged lactose-limited conditions (Zhang and Ferenci, 1999). In contrast, the absence of OmpF results in a large decrease in fitness and these observations are consistent with earlier studies showing decreased growth in porinless mutants (von Meyenburg, 1971). Regulatory mutations causing lack of porin(s) are common in many drug-resistant clinical isolates (Pages et al., 2008; Ferenci and Phan, 2015). Our results suggest that porinless mutants are significantly less competitive at low nutrient levels that may be common in nature. Likewise, the OmpF G119D mutant that constricts the diameter the pore (Jeanteur et al., 1994) and similar structural changes will also result in strongly decreased nutritional fitness.

As determined in studies by Nikaido, the size of the permeant molecule is critical in permitting passage through porin (Nikaido and Nakae, 1979). OmpF permeability is thought to be limited to molecules of molecular weight of less than 600, and rates of permeation are reduced with increasing molecular size. All of the antibiotics used in this study were smaller and wild-type OmpF permitted higher susceptibility to all 9 antibiotics compared with porinless mutant. The most interesting case in terms of size exclusion was with streptomycin, which permeated rapidly despite its high molecular weight (581.6). Even the G119D mutant with restricted fitness permits much-increased streptomycin susceptibility. Our data is inconsistent with the notion that aminoglycoside permeation is porinindependent (Hancock et al., 1991b) because of the OmpF-dependence of kanamycin and streptomycin susceptibility. It has been proposed that the transport of $\beta$-lactams or fluoroquinolones occurs through porins, but involves specific interactions with the porin channel (Nestorovich et al., 2002; Ziervogel and Roux, 2013). The postulated role of binding pockets in permeability is, however, inconsistent with the observed passive diffusion within the concentration range relevant to antibiotic action (Kojima and Nikaido, 2013). Our data do not resolve this question of interactions, but the distinct patterns of susceptibility with different antibiotics suggest distinct paths or antibiotic-channel contacts in OmpF for different permeants. Other environmental factors that influence antibiotic permeability through $\mathrm{OmpF}$ are pH (Cama et al., 2015) or cation concentration (Singh et al., 2012); these environmental properties could also affect patterns of permeability for different antibiotics but were not studied here.

The results in Figure 4 indicate that trade-off shapes are not uniform for structural constraint trade-offs even within a single protein. The strain set had no resource allocation effects, because all strains except the null mutant contained the same amount of porin. The structural constraint variations in the strain set had multiple distinct effects on trade-off geometry. Structural variations can result in accelerating, decelerating or linear trade-off geometries. This result strongly reinforces the plasticity of trade-off shapes, which has already been seen in other studies (Jessup and Bohannan, 2008; Maharjan et al., 2013). This flexibility in shapes does not support the proposal that trade-off geometries can be deduced from fundamental principles of living cells and used to predict stable genetic polymorphisms (Meyer et al., 2015), except perhaps for very constant, narrow niches.

A striking result in Figure 4 is that a small difference in antibiotic structure, even within aminoglycosides or $\beta$-lactams, is sufficient to change a trade-off shape. The most dramatic difference is between spectinomycin (sharply accelerating shape) and other aminoglycosides (both streptomycin and kanamycin have decelerating shapes). The causes of the different shapes can be manifold differences in channel-antibiotic interactions. Porin channels contain numerous charged residues with interactions across the channel (Aguilella-Arzo et al., 2007), so minor variations both in antibiotic net charge, or spacing of charges, could affect not only permeability but also trade-off shape. This is particularly evident in the R42S and R168S substitutions, which both increase fitness on lactose (Figure 2). These two substitutions actually lead to decreased susceptibility specifically with cephalexin for R42S and with ciprofloxacin for R168S. Other antibiotic susceptibilities are not reduced by R42S and R168S, so these residue changes do not have a general exclusion effect. The R42S and R168S results also indicate that mutations in porins contributing to exclusion do not necessarily lead to trade-off costs in terms of fitness.

The shallow slope with nalidixic acid also raises the possibility that decreased susceptibility incurs relatively small fitness differences. This shallow shape is an extension of the accelerating shape over a more extended range of channel change. Exclusion of amoxicillin, nalidixic acid or trimethoprim in ompF mutants can occur with little loss of fitness. Both these trade-off relationships can, for example, result in the evolution of low cost of resistance to sub-lethal concentrations of antibiotic, permitting survival and acquisition of other mutations as occur in most multi-resistant organisms.

Trade-off shapes differ even within the same antibiotic class however. Distinct geometries were exhibited by different aminoglycosides or different $\beta$-lactam antibiotics, so it becomes extremely hard to postulate cost-benefit predictions even amongst related antibiotics. This weakens the general usefulness of determining trade-off shapes as a predictive approach to antibiotic resistance adaptations. The reality is that it is necessary to look at every 
individual antibiotic geometry to decide how tradeoffs influence adaptive outcomes. For example, from the specific shapes in Figure 4, it appears that amoxicillin or trimethoprim exclusion can occur with little loss of fitness. On the other hand, kanamycin and streptomycin exclusion has a high cost. Overall, it is possible to predict outcomes for these particular combinations of situations, but it is impossible to generalise on the costs of antibiotic resistance for antibiotics in all situations. Yet other environmental factors influence antibiotic permeability through OmpF (Singh et al., 2012; Cama et al., 2015) and could affect trade-off shapes.

Perhaps the most important consequence of the demonstrated trade-off plasticity is the difficulty of making generalisations on the predictive usefulness of trade-off shapes. Historically, it has been proposed that knowing the mechanisms of trade-offs and precise shapes can explain ecological behaviour (Stearns, 1989; Tilman, 2000; Hoyle et al., 2008). Indeed, it is undoubtedly the case that particular shapes can be used to predict competitive behaviour (Maharjan et al., 2013; Meyer et al., 2015). On the other hand, previous indications of environmental flexibility in trade-off geometries have been seen and apply to both resource allocation and structural constraint trade-offs (Jessup and Bohannan, 2008; Maharjan et al., 2013). Our results illustrate in the greatest detail yet that trade-offs are exquisitely context-specific. If this high plasticity of shapes generally applies to trade-offs between other pairs of traits, the predictive usefulness of trade-off shapes will be narrowed to highly constrained and unchanging environmental niches.

\section{Conflict of Interest}

The authors declare no conflict of interest.

\section{Acknowledgements}

We thank Ram Maharjan for useful discussions and comments on the manuscript. The research was supported by an Australian Research Council Discovery grant.

\section{References}

Aguilella-Arzo M, Garcia-Celma JJ, Cervera J, Alcaraz A, Aguilella VM. (2007). Electrostatic properties and macroscopic electrodiffusion in $\mathrm{OmpF}$ porin and mutants. Bioelectrochemistry 70: 320-327.

Benson SA, Occi JL, Sampson BA. (1988). Mutations that alter the pore function of the $\mathrm{OmpF}$ porin of Escherichia coli K12. J Mol Biol 203: 961-970.

Blattner FR, Plunkett G, Bloch CA, Perna NT, Burland V, Riley $\mathrm{M}$ et al. (1997). The complete genome squence of Escherichia coli K-12. Science 277: 1453-1462.

Bohannan BJM, Kerr B, Jessup CM, Hughes JB, Sandvik G. (2002). Trade-offs and coexistence in microbial microcosms. Ant van Leeuw Int J G 81: 107-115.
Cama J, Bajaj H, Pagliara S, Maier T, Braun Y, Winterhalter M et al. (2015). Quantification of fluoroquinolone uptake through the outer membrane channel OmpF of Escherichia coli. J Am Chem Soc 137: 13836-13843.

Datsenko KA, Wanner BL. (2000). One-step inactivation of chromosomal genes in Escherichia coli K-12 using PCR products. Proc Natl Acad Sci USA 97: 6640-6645.

De la Cruz MA, Calva E. (2010). The complexities of porin genetic regulation. J Mol Microbiol Biotechnol 18: 24-36.

Death A, Notley L, Ferenci T. (1993). Derepression of LamB protein facilitates outer membrane permeation of carbohydrates into Escherichia coli under conditions of nutrient stress. J Bacteriol 175: 1475-1483.

Delcour AH. (2009). Outer membrane permeability and antibiotic resistance. Biochim Biophys Acta - Prot 1794: 808-816.

Dupont M, James CE, Chevalier J, Pages JM. (2007). An early response to environmental stress involves regulation of $\mathrm{OmpX}$ and $\mathrm{OmpF}$, two enterobacterial outer membrane pore-forming proteins. Antimicrob Agents Chemother 51: 3190-3198.

Dykhuizen DE, Hartl DE. (1983). Selection in chemostats. Microbiol Rev 47: 150-168.

Ferenci T. (2005). Maintaining a healthy SPANC balance through regulatory and mutational adaptation. Mol Microbiol 57: 1-8.

Ferenci T, Phan K. (2015). How porin heterogeneity and trade-offs affect the antibiotic susceptibility of Gramnegative bacteria. Genes 6: 1113-1124.

Ferenci T. (2016). Trade-off mechanisms shaping the diversity of bacteria. Trends Microbiol 24: 209-223.

Fernández L, Hancock REW. (2012). Adaptive and mutational resistance: role of porins and efflux pumps in drug resistance. Clin Microbiol Rev 25: 661-681.

Gudelj I, Weitz JS, Ferenci T, Horner-Devine MC, Marx CJ, Meyer JR et al. (2010). An integrative approach to understanding microbial diversity: from intracellular mechanisms to community structure. Ecol Lett 13: 1073-1084.

Hancock RE, Farmer SW, Li ZS, Poole K. (1991a). Interaction of aminoglycosides with the outer membranes and purified lipopolysaccharide and $\mathrm{OmpF}$ porin of Escherichia coli. Antimicrob Agents Chemother 35: 1309-1314.

Hancock RE, Farmer SW, Li ZS, Poole K. (1991b). Interaction of aminoglycosides with the outer membranes and purified lipopolysaccharide and $\mathrm{OmpF}$ porin of Escherichia coli. Antimicrob Agents Chemother 35: 1309-1314.

Hoyle A, Bowers RG, White A, Boots M. (2008). The influence of trade-off shape on evolutionary behaviour in classical ecological scenarios. J Theor Biol 250: 498-511.

Jeanteur D, Schirmer T, Fourel D, Simonet V, Rummel G, Widmer C et al. (1994). Structural and functional alterations of a colicin-resistant mutant of OmpF porin from Escherichia coli. Proc Natl Acad Sci USA 91: 10675-10679.

Jessup CM, Forde SE, Bohannan BJM. (2005). Microbial experimental systems in ecology. Adv Ecol Res 37: 273-307.

Jessup CM, Bohannan BJM. (2008). The shape of an ecological trade-off varies with environment. Ecol Lett 11: 947-959.

Kneitel JM, Chase JM. (2004). Trade-offs in community ecology: linking spatial scales and species coexistence. Ecol Lett 7: 69-80. 
Kojima S, Nikaido H. (2013). Permeation rates of penicillins indicate that Escherichia coli porins function principally as nonspecific channels. Proc Natl Acad Sci USA 110: E2629-E2634.

Levins R. (1968). Evolution in Changing Environments; Some Theoretical Exploration. Princeton University Press: Princeton, New Jersey, USA.

Lin XM, Wang C, Guo C, Tian YM, Li H, Peng XX. (2012). Differential regulation of OmpC and OmpF by AtpB in Escherichia coil exposed to nalidixic acid and chlortetracycline. J Proteomics 75: 5898-5910.

Liu XQ, Ferenci T. (1998). Regulation of porin-mediated outer membrane permeability by nutrient limitation in Escherichia coli. J Bacteriol 180: 3917-3922.

Maharjan R, Nilsson S, Sung J, Haynes K, Beardmore RE, Hurst LD et al. (2013). The form of a trade-off determines the response to competition. Ecol Lett 16: 1267-1276.

Maloy SR, Nunn WD. (1981). Selection for loss of tetracycline resistance by Escherichia coli. J Bacteriol 145: 1110-1111.

Masi M, Pagès J-M. (2013). Structure, function and regulation of outer membrane proteins involved in drug transport in enterobactericeae: the $\mathrm{OmpF} / \mathrm{C}-$ TolC Case. The Open Microbiology Journal 7: 22-33.

Meyer JR, Gudelj I, Beardmore R. (2015). Biophysical mechanisms that maintain biodiversity through tradeoffs. Nat Commun 6: 6278-6278.

Miller J. (1972). Experiments in Molecular Genetics. Cold Spring Harbor Laboratory: Cold Spring Harbor, NY, USA.

Moreira MAS, Oliveira JA, Teixeira LM, Moraes CA. (2005). Detection of a chloramphenicol efflux system in Escherichia coli isolated from poultry carcass. Vet Microbiol 109: 75-81.

Mortimer PGS, Piddok LJV. (1993). The accumulation of five antibacterial agents in porin-deficient mutants of Escherichia coli. J Antimicrob Chemother 32: 195-213.

Nakae R, Nakae T. (1982). Diffusion of aminoglycoside antibiotics across the outer membrane of Escherichia coli. Antimicrob Agents Chemother 22: 554-559.

Nestorovich EM, Danelon C, Winterhalter M, Bezrukov SM. (2002). Designed to penetrate: Time-resolved interaction of single antibiotic molecules with bacterial pores. Proc Natl Acad Sci USA 99: 9789-9794.

Nikaido H, Nakae T. (1979). The outer membrane of Gramnegative bacteria. Adv Microb Physiol 20: 163-250.

Nikaido H. (2003). Molecular basis of bacterial outer membrane permeability revisited. Microbiol Mol Biol Rev 67: 593-656.

Nikaido H. (2009). Multidrug resistance in bacteria. Annu Rev Biochem 78: 119-146.

Ochs MM, McCusker MP, Bains M, Hancock REW. (1999). Negative regulation of the Pseudomonas aeruginosa outer membrane porin OprD selective for imipenem and basic amino acids. Antimicrob Agents Chemother 43: 1085-1090.
Pages JM, James CE, Winterhalter M. (2008). The porin and the permeating antibiotic: a selective diffusion barrier in Gram-negative bacteria. Nat Rev Microbiol 6: 893-903.

Phale PS, Philippsen A, Widmer C, Phale VP, Rosenbusch JP, Schirmer T. (2001). Role of charged residues at the $\mathrm{OmpF}$ porin channel constriction probed by mutagenesis and simulation. Biochemistry 40: 6319-6325.

Phan K, Ferenci T. (2013). A design-constraint trade-off underpins the diversity in ecologically important traits in species Escherichia coli. ISME J 7: 2034-2043.

Pratt LA, Hsing WH, Gibson KE, Silhavy TJ. (1996). From acids to OsmZ -multiple factors influence the synthesis of the OmpF and OmpC porins in Escherichia coli. Mol Microbiol 20: 911-917.

Saint N, Lou KL, Widmer C, Luckey M, Schirmer T, Rosenbusch JP. (1996). Structural and functional characterization of $\mathrm{OmpF}$ porin mutants selected for larger pore size.2. Functional characterization. J Biol Chem 271: 20676-20680.

Sawai T, Yamaguchi A, Saiki A, Hoshino K. (1992). Ompf channel permeability of quinolones and their comparison with beta-lactams. FEMS Microbiol Lett 95: 105-108.

Simonet V, Mallea M, Pages JM. (2000). Substitutions in the eyelet region disrupt cefepime diffusion through the Escherichia coli OmpF channel. Antimicrob Agents Chemother 44: 311-315.

Singh PR, Ceccarelli M, Lovelle M, Winterhalter M, Mahendran KR. (2012). Antibiotic permeation across the OmpF channel: modulation of the affinity site in the presence of magnesium. J Phys Chem $B$ 116: 4433-4438.

Stearns SC. (1989). Trade-offs in life-history evolution. Funct Ecol 3: 259-268.

Szybalski W, Bryson V. (1952). Genetic studies on microbial cross resistance to toxic agents I.: Cross Resistance of Escherichia coli to Fifteen Antibiotics. J Bacteriol 64: 489-499.

Tilman D. (2000). Causes, consequences and ethics of biodiversity. Nature 405: 208-211.

von Meyenburg K. (1971). Transport-limited growth rates in a mutant of Escherichia coli. J Bacteriol 107: 878888.

Wylie JL, Worobec EA. (1994). Cloning and nucleotide sequence of the Pseudomonas aeruginosa glucoseselective $o p r B$ porin gene and distribution of $\mathrm{OprB}$ within the family Pseudomonadaceae. Eur J Biochem 220: $505-512$.

Zhang E, Ferenci T. (1999). OmpF changes and the complexity of Escherichia coli adaptation to prolonged lactose limitation. FEMS Microbiol Lett 176: 395-401.

Ziervogel BK, Roux B. (2013). The binding of antibiotics in OmpF porin. Structure 21: 76-87.

Supplementary Information accompanies this paper on The ISME Journal website (http://www.nature.com/ismej) 